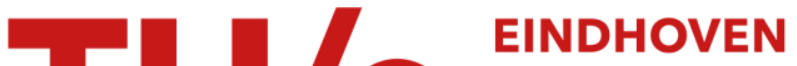

\section{Model order reduction for nonlinear differential algebraic equations in circuit simulation}

\section{Citation for published version (APA):}

Voss, T., Verhoeven, A., Bechtold, T., \& Maten, ter, E. J. W. (2006). Model order reduction for nonlinear differential algebraic equations in circuit simulation. (CASA-report; Vol. 0637). Technische Universiteit Eindhoven.

\section{Document status and date:}

Published: 01/01/2006

\section{Document Version:}

Publisher's PDF, also known as Version of Record (includes final page, issue and volume numbers)

\section{Please check the document version of this publication:}

- A submitted manuscript is the version of the article upon submission and before peer-review. There can be important differences between the submitted version and the official published version of record. People interested in the research are advised to contact the author for the final version of the publication, or visit the $\mathrm{DOI}$ to the publisher's website.

- The final author version and the galley proof are versions of the publication after peer review.

- The final published version features the final layout of the paper including the volume, issue and page numbers.

Link to publication

\section{General rights}

Copyright and moral rights for the publications made accessible in the public portal are retained by the authors and/or other copyright owners and it is a condition of accessing publications that users recognise and abide by the legal requirements associated with these rights.

- Users may download and print one copy of any publication from the public portal for the purpose of private study or research.

- You may not further distribute the material or use it for any profit-making activity or commercial gain

- You may freely distribute the URL identifying the publication in the public portal.

If the publication is distributed under the terms of Article 25fa of the Dutch Copyright Act, indicated by the "Taverne" license above, please follow below link for the End User Agreement:

www.tue.nl/taverne

Take down policy

If you believe that this document breaches copyright please contact us at:

openaccess@tue.nl

providing details and we will investigate your claim. 


\section{EINDHOVEN UNIVERSITY OF TECHNOLOGY}

Department of Mathematics and Computer Science

\section{CASA-Report $06-37$}

November 2006

Model order reduction for nonlinear differential algebraic equations in circuit simulation

by

T. Voss, A. Verhoeven, T. Bechtold, E.J.W. ter Maten

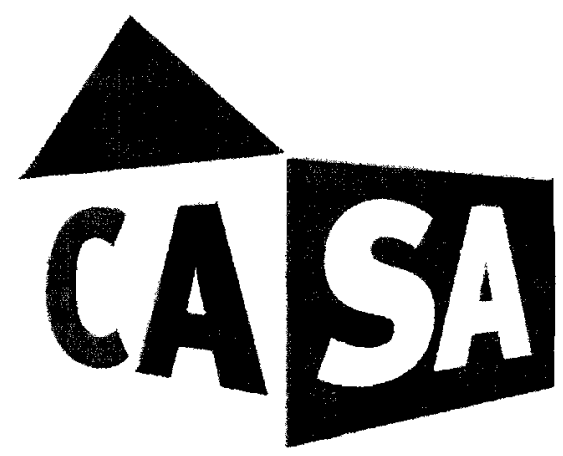

Centre for Analysis, Scientific computing and Applications

Department of Mathematics and Computer Science

Eindhoven University of Technology

P.O. Box 513

5600 MB Eindhoven, The Netherlands

ISSN: 0926-4507 


\title{
Model Order Reduction for Nonlinear Differential Algebraic Equations in Circuit Simulation.
}

\author{
Thomas Voss ${ }^{3}$, Arie Verhoeven ${ }^{12}$, Tamara Bechtold ${ }^{1}$, and Jan ter Maten ${ }^{1}$ \\ 1 NXP Semiconductors tamara.bechtoldQphilips.com \\ 2 Eindhoven University of Technology averhoev@rin.tue.nl \\ ${ }^{3}$ Delft University of Technology t.voss@tudelft.nl
}

Summary. In this paper we demonstrate model order reduction of a nonlinear academic model of a diode chain. Two reduction methods, which are suitable for nonlinear differential algebraic equation systems are used, the trajectory piecewise linear approach and the proper orthogonal decomposition with missing point estimation.

\section{Introduction}

The dynamics of electrical circuits at time $t$ can be generally described by the nonlinear, first order, differential-algebraic equation (DAE) system of the form:

$$
\frac{d}{d t} \mathbf{q}(\mathbf{x})+\mathbf{j}(\mathbf{x})+B \mathbf{u}(t)=\mathbf{0}
$$

where $\mathbf{x} \in \mathbb{R}^{n}$ represents the unknown vector of circuit variables in time $t$, the vector-valued functions $\mathbf{q}, \mathbf{j}: \mathbb{R} \times \mathbb{R}^{n} \rightarrow \mathbb{R}^{n}$ represent the contributions of, respectively, reactive elements (such as capacitors and inductors) and of nonreactive elements (such as resistors) and $B \in \mathbb{R}^{n \times m}$ is the distribution matrix for the excitation vector $\mathbf{u}: \mathbb{R} \rightarrow \mathbb{R}^{m}$. There are several established methods, such as sparse-tableau, modified nodal analysis etc. which generate the system (1) from the netlist description of electrical circuit. The dimension $n$ of (1) is of the order of the number of elements in the circuit, which means that it can be extremely large, as today's VLSI circuits have hundreds of millions of elements.

Mathematical model order reduction (MOR) aims to replace (1) by a system of much smaller dimension, which can be solved by suitable DAE solvers within acceptable time. At present, however, only linear MOR techniques are well-enough developed and properly understood to be employed [1]. To that end, we either linearise the system (1) or decouple it into nonlinear and linear subcircuits (interconnect macromodeling or parasitic subcircuits 
[2]). The nonlinear MOR techniques are less developed and less understood than the linear ones. In this paper we present the application of two most promising nonlinear reduction methods on an academic diode chain model. These are the trajectory piecewise linear approach (TPWL) [3] and the proper orthogonal decomposition (POD) [4] supported by missing point estimation (MPE) technique [5].

\section{Trajectory Piecewise Linear Model Order Reduction}

The idea behind the TPWL method is to linearise (1) several times along a training trajectory (corresponding to some typical input). The local systems are then used to create a global reduced subspace. The final TPWL model is constructed as a weighted sum of all local linearised reduced systems.

\subsection{Creating the local linearised models}

The disadvantage of standard linearisation methods is that they deliver good results, only in the surrounding of the chosen linearisation tuple (LT) $\left(x\left(t_{i}\right), t_{i}\right)$. To overcome this, in TPWL approach several linearised models are created. This guarantees the quality of the results whenever the solution stays close to one of the chosen LTs. The procedure for selection of LTs can be described by the following steps:

1. Set an absolute accuracy factor $\varepsilon>0$, set $i=1$.

2. Linearise the system around the $i$-th LT $\left(x_{i}, t_{i}\right)$. This implies:

$$
C_{i} \dot{\mathbf{x}}+G_{i} \mathbf{x}+B_{i} \mathbf{u}(t)=0
$$

with $C_{i}=\left.\frac{\partial}{\partial x} \mathbf{q}(t, \mathbf{x})\right|_{\mathbf{x}_{i}, t_{i}}$ and $G_{i}=\left.\frac{\partial}{\partial x} \mathbf{j}(t, \mathbf{x})\right|_{\mathbf{x}_{i}, t_{i}}$, where $x_{i}$ stays for $x\left(t_{i}\right)$. Save $C_{i}, G_{i}$ and $B_{i}$.

3. Reduce the linearised system to dimension $r \ll n$ with an appropriate linear MOR method, like "Poor Man's TBR" [6] or Krylov-subspace methods [7]. This implies:

$$
C_{i}^{r} \dot{\mathbf{z}}+G_{i}^{r} \mathbf{z}+B_{i}^{r} \mathbf{u}(t)=0
$$

where $C_{i}^{r}=V_{i}^{\top} C_{i} V, G_{i}^{r}=V_{i}^{\top} G_{i} V_{i}, B_{i}^{r}=V_{i}^{\top} B$ with $V_{i} \in \mathbb{R}^{n \times r}, \mathbf{z} \in \mathbb{R}^{r}$ and $\mathrm{x} \approx V_{i} \mathrm{z}$. Save the local projection matrix $V_{i}$.

4. Integrate both, the reduced system (3) and the original system (1) choosing the same time-steps $t_{k}$. When $\frac{\left\|V_{i} \mathbf{z}\left(t_{k}\right)-\mathbf{x}\left(t_{k}\right)\right\|}{\left\|\mathbf{x}\left(t_{k}\right)\right\|}>\varepsilon$ chose $\left(\mathbf{x}\left(t_{k}\right), t_{k}\right)$ as $i+1$-th LT . Set $i=i+1$. Go to step 2 .

The steps 2 to 4 are repeated until the end of the given trajectory has been reached. In this way, $s$ local reduced subspaces with bases $V_{1}, \ldots, V_{s}$ are created. 


\subsection{Creating the global reduced subspace}

All local reduced subspaces are merged into the global reduced subspace and each local linearised system (2) is now projected onto this global subspace. The procedure can be described by the following steps:

1. Define $\tilde{V}=\left[V_{1}, \ldots, V_{s}\right]$.

2. Calculate the SVD of $\tilde{V}: \tilde{V}=U \Sigma W^{\top}$ with $U=\left[u_{1}, \ldots, u_{n}\right] \in \mathbb{R}^{n}, \Sigma \in$ $\mathbb{R}^{n \times r s}$ and $W \in \mathbb{R}^{r s \times r s}$.

3. Define new global projection matrix $V_{g}$ as $\left[u_{1}, \ldots, u_{r}\right]$.

4. Project each local linearised system (2) onto $V_{g}$.

\subsection{Creating the TPWL model by weighting}

All local reduced linearised reduced systems are combined in a weighted sum to build the global TPWL model:

$$
\sum_{i=1}^{s} w_{i} V_{g}^{\top} C_{i} V_{g} \dot{\mathbf{z}}+\sum_{i=1}^{s} w_{i} V_{g}^{\top} G_{i} V_{g} \mathbf{z}+\sum_{i=1}^{s} w_{i} V_{g}^{\top} B_{i} \mathbf{u}(t)=0
$$

A weight $w_{i}$ determines the influence of the $i$-th local system to the global system. The weights can be chosen by making them distance depending, which means that $w_{i}$ is chosen large if the solution $\mathbf{z}$ of (??) is close to the $i$-th LT, else the weight should be small. For more details on how to chose weights, see [8].

\section{Proper Orthogonal Decomposition combined with Missing Point Estimation}

The idea behind POD is to directly project the original nonlinear system (1) onto some subspace with smaller dimension. As this, however, does not lead to the reduction of the computational time, MPE is used to speed up the simulation.

\section{1 "Classical" POD}

The POD projection basis $V_{P O D}$ is an orthonormal basis, which is derived from the collected "snapshots" at the time points $t_{i}$ :

$$
X=\left[\mathbf{x}\left(t_{1}\right) \ldots \mathbf{x}\left(t_{s}\right)\right]
$$

The POD basis is found from the SVD of $X: X=U \Sigma W^{\top}$ with $U=$ $\left[u_{1}, \ldots, u_{n}\right] \in \mathbb{R}^{n}, \Sigma \in \mathbb{R}^{n \times s}$ and $W \in \mathbb{R}^{s}$, as $V_{P O D}=\left[u_{1}, \ldots, u_{r}\right]$ with $r \ll n$. Finally the original system is replaced by the following Galerkin projection

$$
\frac{d}{d t} V_{P O D}^{\top} \mathbf{q}\left(V_{P O D} \mathbf{z}\right)+V_{P O D}^{\top} \mathbf{j}\left(V_{P O D} \mathbf{z}\right)+V_{P O D}^{\top} B \mathbf{u}(t)=0
$$




\subsection{Missing Point Estimation}

In the projection schemes, usually the original numerical model is projected onto the chosen subspace. In the case of linear systems, i. e. when $\mathbf{q}(\mathbf{x})=C \mathbf{x}$ and $\mathbf{j}(\mathbf{x})=G \mathbf{x}$ the projections $V_{P O D}^{T} \mathbf{q}\left(V_{P O D} \mathbf{z}\right)$ and $V_{P O D}^{T} \mathbf{j}\left(V_{P O D} \mathbf{z}\right)$ can be computed "in advance" and will deliver the matrices of the reduced system (as in (3)). For the nonlinear systems however, the projection requires the complete evaluations of $q$ and $\mathbf{j}$ and hence, the solution of (6) will not be faster than the solution of (1). In order to speed it up, a so called missing point estimation can be applied. Assume that:

$$
V_{P O D} \approx P^{\top} P \tilde{V}_{P O D},
$$

where $P \in\{0,1\}^{g \times n}$ is a selection matrix with $P P^{\top}=I_{g}$. Now introduce the restricted basis $V_{M P E}=P \tilde{V}_{P O D}$. Then $V_{P O D}^{T} \mathbf{q}\left(V_{P O D} \mathbf{z}\right) \approx$ $\tilde{V}_{P O D}^{T} P^{\top} P \mathbf{q}\left(P^{\top} P \tilde{V}_{P O D} \mathbf{z}\right)=V_{M P E}^{T} P \mathbf{q}\left(P^{\top} V_{M P E} \mathbf{z}\right)$ and similar for $j$. Hence, only $g$ elements of $\mathbf{q}$ and $\mathbf{x}$ have to be evaluated, which is much cheaper than evaluating $\mathbf{q}$ and $\mathbf{j}$ if $g<<n$.

We use an iterative version of the greedy algorithm [5] in order to find a selection matrix $P$ with minimal dimension $g$, such that

$$
\operatorname{cond}\left(V_{P O D}^{\top} P^{\top} P V_{P O D}\right)<\mathrm{TOL}
$$

is fullfiled.

\section{Numerical results}

We considered the academic diode chain model shown in Fig. 1, which is described through the following equations:

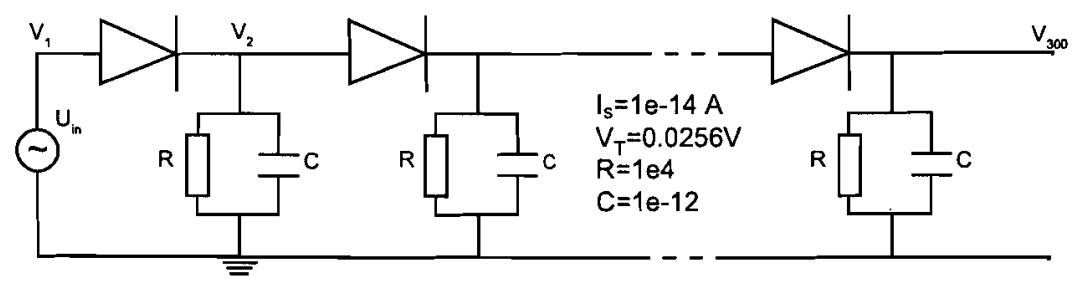

Fig. 1. Structure of the test circuit

$$
\begin{aligned}
V_{1}-U_{\text {in }}\left(10^{9} t\right) & =0, \\
i_{E}-g\left(V_{1}, V_{2}\right) & =0,
\end{aligned} \quad\left\{\begin{array}{rl}
g\left(V_{a}, V_{b}\right)= \\
g\left(V_{1}, V_{2}\right)-g\left(V_{2}, V_{3}\right)-C \dot{V}_{2}-\frac{1}{R} V_{2} & =0, \\
\vdots & \\
g\left(V_{N-1}, V_{N}\right)-g\left(V_{N}, V_{N+1}\right)-C \dot{V}_{N}-\frac{1}{R} V_{N} & =0,-1) \text { if } V_{a}-V_{b}>0.5 \\
0 & \text { otherwise } \\
g\left(V_{N}, V_{N+1}\right)-C \dot{V}_{N+1}-\frac{1}{R} V_{N+1} & =0,
\end{array} \quad U_{\text {in }}(t)=\left\{\begin{array}{cc}
20 & \text { if } t \leq 10 \\
170-15 t & \text { if } 10<t \leq 11 \\
5 & \text { if } t>11
\end{array}\right.\right.
$$



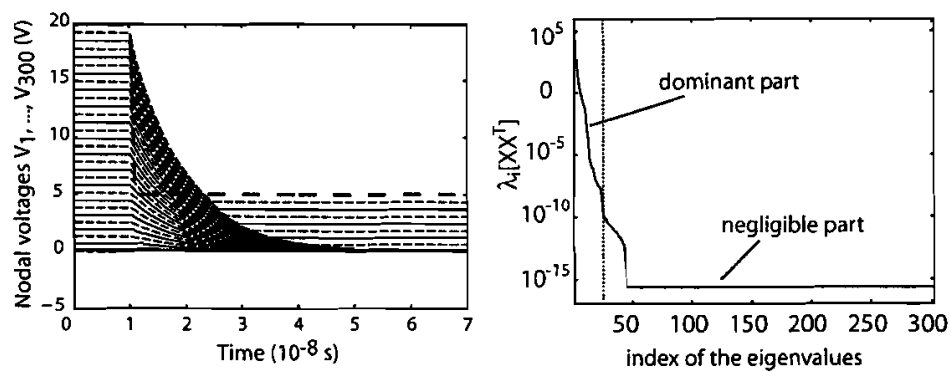

Fig. 2. Numerical solution of the full-scale nonlinear diode chain model (left) and the eigenvalues of the correlation matrix $\frac{1}{n} X X^{T}$ (right).

Fig. 2 (left) shows the numerical solution (nodal voltage in each node) of the original model, computed by the Euler Backward method with fixed step sizes of $0.1 \mathrm{~ns}$. It further indicates (right) the redundancy of the model, as most of the eigenvalues of the correlation matrix $\frac{1}{n} X X^{T}$ can be neglected. Figure 3 shows the relative errors over all nodes in the time interval $[0,70 \mathrm{~ns}]$, defined as $\varepsilon_{r}=\frac{\|V \mathbf{z}-\mathbf{x}\|}{\|\mathbf{x}\|}$, for the reduced models of different orders constructed by TPWL (left) and POD (right). For TPWL the relative error is most of the time lower then the chosen error bound $\varepsilon=0.025$. Furthermore, for higher order reduced models, a smaller number of LTs has been used than for the reduced models with lower order, as the local systems with higher orders are more accurate. E.g. for a reduced model of order 100 we have used $42 \mathrm{LTs}$ and for smaller reduced models $60 \mathrm{LTs}$. The POD models are, as expected, more accurate, but much slower to simulate than the TPWL models (see the corresponding extraction and simulation times in Table 1). A significant speed up has been achieved by combining the POD with MPE.
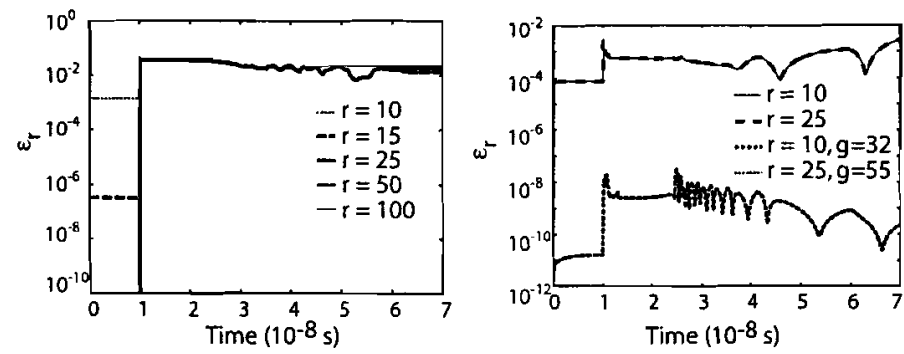

Fig. 3. Relative errors over all nodes for the reduced models created by TPWL (left) and by POD (right). 
Thomas Voss, Arie Verhoeven, Tamara Bechtold, and Jan ter Maten

Table 1. Comparison of extraction and simulation times in seconds.

\begin{tabular}{l|l|l|l||l|l|l|l|l|l|l|l|l|} 
Model & $r$ & $\mid$ Extr. time & Sim. time & Model & $|r| g \mid$ Extr. time & Sim. time \\
\hline Original & 302 & 0 & 142 & POD & $10 \mid$ & 302 & 142 & 168 \\
TPWL & 10 & 290 & 1.1 & POD & 25 & 302 & 142 & 182 \\
TPWL & 25 & 285 & 1.5 & POD + MPE & 10 & 32 & 146 & 74 \\
TPWL & 50 & 206 & 2.3 & POD + MPE & 25 & 55 & 151 & 123
\end{tabular}

\section{Conclusion and outlook}

The TPWL method seems to be a promising technique to reduce the simulation time for nonlinear DAE systems. It's main advantage is the application of well-developed linear model reduction techniques. The POD method delivers reduced models which are more accurate but also much more expensive to compute. Hence, the missing point estimation is necessary to achieve a reduction of simulation time at all. Both techniques offer a good starting point for further research on MOR of non-linear dynamical systems.

Finally, we would like to thank Dr. B. Tasić for his help with the diode chain model and to acknowledge the EU support through the COMSON RTN project.

\section{References}

1. Antoulas, A. C.: Approximation of Large-Scale Dynamical Systems. Society for Industrial and Applied Mathematics, (2005)

2. Freund, R. W.: Krylov-subspace methods for reduced order modeling in circuit simulation. Journal of Computational and Applied Mathematics, Vol. 123, pp. 395-421,(2000)

3. Rewienski, M., White, J.: A Trajectory Piecewise-Linear Approach to Model Order Reduction and Fast Simulation of Nonlinear Circuits and Micromachined Devices. In: Proc. of the Int. Conf. on CAD, 252-7 (2001)

4. Astrid, P.: Reduction of process simulation models: a proper orthogonal decomposition approach. PhD dissertation, Eindhoven University of Technology, Department of Electrical Engineering, (2004)

5. Astrid, P., Verhoeven, A.: Application of Least Squares MPE technique in the reduced order modeling of electrical circuits. In: Proceedings MTNS, Kyoto (2006)

6. Phillips, J., Silvera, L.M.: Poor Man's TBR: A simple model reduction scheme, IEEE transactions on computer-aided design of integrated circuits and systems. Vol. 14 No. 1, (2005)

7. Odabasioglu, A., Celik, M., Pileggi, L.T.: PRIMA: Passive Reducedorder Interconnect Macromodeling Algorithm, IEEE Transactions on Computer-Aided Design of Integrated Circuits and Systems, Vol. 17, No. 8, 645-654, (1998)

8. Voss, T.: Model reduction for nonlinear differential algebraic equations, M. Sc. Thesis, University of Wupertal, (2005) Journal of Computational and Applied Mathematics, Vol. 123, pp. 395-421,(2000) 